KEK-TH-1178

OIQP- $07-11$

YITP-07-56

\title{
Hawking Radiation via Higher-spin Gauge Anomalies
}

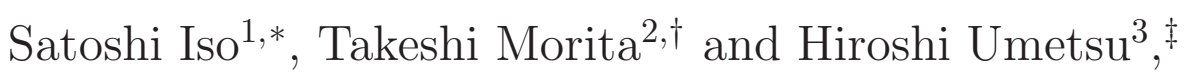 \\ ${ }^{1}$ Institute of Particle and Nuclear Studies \\ High Energy Accelerator Research Organization (KEK) \\ Oho 1-1, Tsukuba, Ibaraki 305-0801, Japan \\ ${ }^{2}$ Yukawa Institute for Theoretical Physics, Kyoto University \\ Kyoto 606-8502, Japan \\ ${ }^{3}$ Okayama Institute for Quantum Physics \\ Kyoyama 1-9-1, Okayama 700-0015, Japan
}

\begin{abstract}
We give a higher-spin generalization of the anomaly method for the Hawking radiation from black holes. In the paper [13] higher-spin generalizations of the gauge (and gravitational) anomalies in $\mathrm{d}=2$ were obtained. By applying these anomalies to black hole physics, we derive the higher moments of the Hawking fluxes. We also give a higher-spin generalization of the trace anomaly method by Christensen and Fulling [12].
\end{abstract}

*satoshi.iso@kek.jp

†mtakeshi@yukawa.kyoto-u.ac.jp, takeshi@theory.tifr.res.in,

Present address: Department of Theoretical Physics, Tata Institute of Fundamental Research, Homi Bhabha Road, Mumbai 400005, India.

${ }^{\ddagger}$ hiroshi_umetsu@pref . okayama.jp 


\section{Introduction}

Hawking radiation is a universal quantum effect which arises in the background spacetime with event horizons [1, 2]. The radiation is essentially originated from the causal structure of the event horizon and the quantum behavior of fields around it. Such universal behavior is emphasized in the recent derivation of Hawking radiation based on gravitational anomaly [3]. One of the key observations is that near the horizon, due to the infinite blue shift, ordinary matter fields in the black hole backgrounds behave as if they are an infinite set of massless two-dimensional fields, and the causal structure of the horizon makes the two-dimensional fields effectively chiral at the horizon. i.e. the ingoing modes (which can be interpreted as left moving modes) can be classically decoupled from the outside region of the horizon. Chiral theories suffer from gravitational anomalies. Since the underlying theories must be covariant, they should be cancelled by the quantum effect of classically irrelevant ingoing modes at the horizon. The requirement of the anomaly cancellation leads to the existence of the Hawking energy-flux from the black hole in the Unruh vacuum [4]. This method was, after correcting some errors in the original procedure*, generalized to charged black holes [5], and further applied to rotating black holes [6, 7] and others [8, 9].

The anomaly method, so far, could obtain only partial information of Hawking radiation because the gravitational and gauge anomalies contain only the information of fluxes of energy and charge. These fluxes correspond to the zero-th and first moments of the thermal distribution of radiation, and in order to obtain the full information, we need to calculate all the other higher moments. In [10], by using conformal field theory technique, we calculated fluxes of higher-spin (HS) currents in a Schwarzschild black hole. They correspond to the higher-moments of Hawking radiation and the complete thermal distribution is reproduced. In this calculation we have used transformation properties of HS currents under conformal transformations, which relate quantities near the horizon with those at infinity. This method was further generalized to charged black holes by considering gauge transformations in addition to the conformal transformations [11]. It is, however, not a direct generalization of the anomaly method and the relation to the previous approaches such as the anomaly method [3, 5] or the method by Christen and Fulling [12] was not clear.

\footnotetext{
*It is explained in the appendix [8] why the original procedure in [3] does not work for charged or rotating black holes
} 
In this paper, by using the generalizations of gauge (and gravitational) anomalies in $d=2$ [13], we show that we can reproduce the higher-spin fluxes of Hawking radiation for spin 3 and spin 4 currents constructed from fermions. This is the direct generalization of the anomaly method [3, 5]. We also show that by solving the conservation equations and trace anomalies for spin 3 and 4 currents, we can again reproduce the same fluxes. It is a HS generalization of the trace anomaly method by Christensen-Fulling [12]. These

calculations can be straightforwardly extended to higher spins than 4, but they become very complicated. Instead of it, we show how we can obtain the asymptotic and nearhorizon behavior of HS currents by using the results in [13].

The organization of the paper is as follows. In section 2, we first summarize the results of higher-spin gauge and trace anomalies obtained in [13. In section 3, we use the results of HS gauge anomalies (up to spin 4) to reproduce the higher spin fluxes of Hawking radiation. Then in section 4, we give a direct generalization of the method by Christensen and Fulling. Finally, by using the relations between holomorphic currents and covariant currents in [13], we reproduce the asymptotic and near horizon behavior of the HS currents, and obtain the full thermal spectrum of Hawking radiation. These derivations clarify some points which were obscure in the previous papers [10, 11].

\section{Higher-spin gauge and trace anomalies}

In this section, we briefly summarize anomalies for higher-spin currents obtained in [13]. We consider a two-dimensional system with $c_{L}$ left-handed and $c_{R}$ right-handed fermions in the electric and gravitational backgrounds. If $c_{L} \neq c_{R}$, the gauge and the general coordinate invariance are broken due to the gauge and gravitational anomalies. In the paper [13], we showed that the higher-spin gauge currents constructed from the fermions also receive quantum anomalies in such chiral theories. Since we are here interested in the application to the Hawking radiation from charged black holes, it is sufficient to know higher-spin generalizations of the gauge (or gravitational) anomalies in the electric and the gravitational backgrounds, i.e. no HS gauge field backgrounds are necessary.

For the $U(1)$ current, it is well known that the gauge and chiral anomalies are given 
by

$$
\begin{aligned}
\nabla_{\mu} J^{\mu} & =-\frac{\left(c_{R}-c_{L}\right)}{2} \frac{\hbar}{2 \pi} \epsilon^{\mu \nu} F_{\mu \nu}, \\
\nabla_{\mu} J^{5 \mu} & =\frac{\left(c_{L}+c_{R}\right)}{2} \frac{\hbar}{2 \pi} \epsilon^{\mu \nu} F_{\mu \nu} .
\end{aligned}
$$

(2.1) is the gauge anomaly of the covariant current in $\mathrm{d}=2$.

In the case of the energy-momentum (EM) tensor, we have the gravitational and trace anomalies,

$$
\begin{aligned}
\nabla^{\mu} T_{\mu \nu} & =F_{\mu \nu} J^{\mu}-\frac{\hbar}{48 \pi} \frac{c_{R}-c_{L}}{2} \epsilon_{\mu \nu} \nabla^{\mu} R \\
T_{\mu}^{\mu} & =\frac{\hbar}{24 \pi} \frac{c_{L}+c_{R}}{2} R .
\end{aligned}
$$

This is the covariant form of the gravitational anomaly for the covariant EM tensor. The first term of the r.h.s. in (2.3) is classical violation of the matter energy momentum tensor in the electric background by the coupling between the charge current $J$ and the electric field $E$. This term arises because the electric background transforms under the general coordinate transformations.

These anomalies for the $U(1)$ current and the EM tensor are usually calculated by the diagrammatic method or in the path integral formulation [14, 15], but in $\mathrm{d}=2$ they can be also calculated easily by using conformal field theory technique. In [13], we generalized this CFT method to calculate anomalies for spin 3 and 4 currents constructed from fermions.

In the case of spin 3 current, the corresponding gauge and trace anomalies are given by

$$
\begin{aligned}
\nabla_{\mu} J_{\nu \rho}^{(3) \mu}= & -F_{\nu \mu} J_{\rho}^{(2) \mu}-F_{\rho \mu} J_{\nu}^{(2) \mu}-\frac{1}{16} \nabla_{\nu}\left(R J_{\rho}^{(1)}\right)-\frac{1}{16} \nabla_{\rho}\left(R J_{\nu}^{(1)}\right)+\frac{1}{16} g_{\nu \rho} \nabla_{\mu}\left(R J^{(1) \mu}\right) \\
& +\frac{\hbar}{48 \pi} \frac{c_{R}-c_{L}}{2}\left(\epsilon_{\nu \sigma} \nabla^{\sigma} \nabla_{\mu} F_{\rho}^{\mu}+\epsilon_{\rho \sigma} \nabla^{\sigma} \nabla_{\mu} F^{\mu}{ }_{\nu}-g_{\nu \rho} \epsilon_{\alpha \sigma} \nabla^{\sigma} \nabla_{\mu} F^{\mu \alpha}\right) \\
J^{(3) \mu}= & \frac{\hbar}{12 \pi} \frac{c_{L}+c_{R}}{2} \nabla_{\mu} F_{\nu}^{\mu} .
\end{aligned}
$$

See [13] for the details of the definitions of the spin 3 (and 4) currents, and the derivations of the anomalies. The first equation is the spin 3 generalization of the covariant form of gauge (or gravitational) anomaly while the second is that of the trace anomaly. The r.h.s. of the first equation consists of classical and quantum parts. The classical parts show that the electric and the gravitational backgrounds are not invariant under gauge transformations associated with the spin 3 currents. The quantum part is the spin 3 generalization of the gauge anomalies in the electric and gravitational backgrounds. 
For spin 4 current, they are given by

$$
\begin{aligned}
\nabla^{\mu} J_{\mu \nu \rho \sigma}^{(4)}= & F_{\mu \nu} J_{\rho \sigma}^{(3) \mu}+F_{\mu \rho} J_{\sigma \nu}^{(3) \mu}+F_{\mu \sigma} J_{\nu \rho}^{(3) \mu}-\frac{1}{8} R\left(\nabla_{\nu} J_{\rho \sigma}^{(2)}+\nabla_{\rho} J_{\sigma \nu}^{(2)}+\nabla_{\sigma} J_{\nu \rho}^{(2)}\right) \\
& -\frac{1}{6}\left(J_{\nu \rho}^{(2)} \nabla_{\sigma} R+J_{\rho \sigma}^{(2)} \nabla_{\nu} R+J_{\sigma \nu}^{(2)} \nabla_{\rho} R\right) \\
& -\frac{1}{24}\left(J_{\nu}^{(1)} \nabla_{\rho} \nabla_{\mu} F_{\sigma}^{\mu}+J_{\rho}^{(1)} \nabla_{\sigma} \nabla_{\mu} F_{\nu}^{\mu}+J_{\sigma}^{(1)} \nabla_{\nu} \nabla_{\mu} F_{\rho}^{\mu}\right. \\
& \left.+J_{\rho}^{(1)} \nabla_{\nu} \nabla_{\mu} F_{\sigma}^{\mu}+J_{\nu}^{(1)} \nabla_{\sigma} \nabla_{\mu} F_{\rho}^{\mu}+J_{\sigma}^{(1)} \nabla_{\rho} \nabla_{\mu} F^{\mu}{ }_{\nu}\right) \\
& -\frac{\hbar}{960 \pi} \frac{c_{R}-c_{L}}{2}\left(\epsilon_{\nu \alpha} \nabla^{\alpha} \nabla_{\rho} \nabla_{\sigma} R+\epsilon_{\rho \alpha} \nabla^{\alpha} \nabla_{\sigma} \nabla_{\nu} R+\epsilon_{\sigma \alpha} \nabla^{\alpha} \nabla_{\nu} \nabla_{\rho} R\right) \\
& -\frac{1}{4}\left(g_{\nu \rho} \hat{C}_{\sigma}+g_{\rho \sigma} \hat{C}_{\nu}+g_{\sigma \nu} \hat{C}_{\rho}\right), \\
J_{\mu \nu \rho}^{(4) \mu}= & -\frac{\hbar}{160 \pi} \frac{c_{L}+c_{R}}{2} \nabla_{\nu} \nabla_{\rho} R+g_{\nu \rho} \frac{c_{L}+c_{R}}{2}\left[\frac{\hbar}{160 \pi} \nabla^{2} R+\frac{\hbar}{24 \pi}\left(\tilde{F}^{2}-\frac{13}{120} R^{2}\right)\right]
\end{aligned}
$$

where $\hat{C}_{\nu}$ is defined as

$$
\begin{aligned}
\hat{C}_{\nu} \equiv & -\frac{1}{4} R \nabla_{\rho} J_{\nu}^{(2) \rho}-\frac{1}{3} J_{\nu}^{(2) \rho} \nabla_{\rho} R-\frac{1}{12}\left(J^{(1) \rho} \nabla_{\rho} \nabla_{\mu} F_{\nu}^{\mu}+J_{\rho}^{(1)} \nabla_{\nu} \nabla_{\mu} F^{\mu \rho}\right) \\
& -\frac{\hbar}{960 \pi} \frac{c_{R}-c_{L}}{2}\left(\epsilon_{\nu \alpha} \nabla^{\alpha} \nabla_{\rho} \nabla^{\rho} R+2 \epsilon_{\rho \alpha} \nabla^{\alpha} \nabla^{\rho} \nabla_{\nu} R\right) .
\end{aligned}
$$

Here we notice that these forms of anomalies are specific to fermions. In the case of bosons, or fields with different conformal weights, they have different forms.

\section{Anomaly method for Hawking radiation}

We now apply our results summarized in the previous section to Hawking radiation in black hole backgrounds. In black hole backgrounds, by using an appropriate harmonic expansion, one can describe matter fields near the horizon by an infinite set of twodimensional fields in the $(t, r)$ section of spacetime. Further since mass and potential terms are suppressed in the near-horizon region and kinetic terms dominate the action there, the theory becomes conformal near the horizon [16] and we can employ conformal field theory techniques. Hence the following analysis of Hawking radiation can be applied to any dimensional black holes, not restricted to $\mathrm{d}=2$.

In [10], we derived fluxes of the HS currents in a Schwarzschild black hole and showed that they coincide with the higher moments of the thermal distribution. We there used 
transformation properties of the holomorphic currents under a conformal transformation which relate quantities near the horizon with those at infinity, and imposed the regularity condition at the horizon. Further this analysis was extended to charged black holes by considering gauge transformations in addition to conformal transformations [11]. The above method can successfully reproduce the thermal radiation with the correct Hawking temperature, but it will be instructive to obtain the same results by direct applications of the anomaly equations in the previous section. In this section, we give a direct generalization of the anomaly method [3, 5] to spin 3 and 4 fluxes.

In the paper [3], only the consistent form of the energy-momentum tensor is used, but in [5], we showed that the boundary condition at the horizon should be imposed for the covariant current. See the appendix of [6] for why the boundary condition employed in [3] does not work for the charged black holes. In the following discussions, we adopt the method using only the covariant currents [17] because the anomaly equations in the previous section are written in the covariant form and we do not discuss the consistent currents.

\subsection{Spin 3 gauge current}

First let us consider the spin 3 current. We divide the region outside the horizon into near-horizon region $H\left(r \in\left[r_{H}, r_{+}+\epsilon\right]\right)$ and the other region $O\left(r>r_{+}+\epsilon\right)$. If we omit the ingoing modes near the horizon, the currents have the anomalies there. Thus the covariant current $J_{(H) \nu \rho}^{(3) \mu}$ in the near-horizon region satisfies eq. (2.5) with $c_{L}=0, c_{R}=1$. In the following, we put $\hbar=1$ for notational simplicity. $\hbar$ can be recovered by replacing $(1 / \pi)$ by $(\hbar / \pi)$ in the anomaly equations.

Since the expectation value of the current depends only on $r$ in the black hole background considered here, the $\nu=\rho=t$ component of the equation (2.5) becomes

$$
\begin{aligned}
\left\langle\partial_{r} J_{(H) t t}^{(3) r}\right\rangle= & -2 F_{t r}\left\langle J_{(H){ }_{t}}^{(2) r}\right\rangle-\frac{1}{8} \nabla_{t}\left(R\left\langle J_{(H) t}^{(1)}\right\rangle\right)+\frac{1}{16} g_{t t} \nabla_{\mu}\left(R\left\langle J_{(H)}^{(1) \mu}\right\rangle\right) \\
& +\frac{1}{98 \pi}\left(-2 \nabla^{r} \nabla_{\mu} F^{\mu}{ }_{t}-g_{t t} \epsilon_{\alpha \sigma} \nabla^{\sigma} \nabla_{\mu} F^{\mu \alpha}\right) .
\end{aligned}
$$

Here $\left\langle J_{(H)}^{(1) r}\right\rangle$ and $\left\langle J_{(H) t}^{(2) r}\right\rangle$ are expectation values of the covariant $U(1)$ current and the covariant EM tensor in the near-horizon region, respectively. They are determined by solving eqs. (2.1) and (2.3) with $c_{L}=0, c_{R}=1$ and requiring that they vanish at the 
horizon. The results are

$$
\begin{aligned}
\left\langle J_{(H)}^{(1) r}\right\rangle & =\frac{1}{2 \pi}\left(A_{t}(r)-A_{t}\left(r_{+}\right)\right) \\
\left\langle J_{(H) t}^{(2) r}\right\rangle & =\int_{r_{+}}^{r} d r \partial_{r}\left[-\frac{1}{2 \pi} A_{t}\left(r_{+}\right) A_{t}(r)+\frac{1}{4 \pi} A_{t}(r)^{2}+\frac{1}{96 \pi}\left(f f^{\prime \prime}-\frac{1}{2} f^{\prime 2}\right)\right] .
\end{aligned}
$$

See [5] and [17] for the details.

Eq. (3.1) can be solved by using the above solutions for spin 1 and 2 currents and imposing the vanishing condition at the horizon, $\left\langle J_{(H)}^{(3) r}\left(r_{+}\right)\right\rangle=0$. This vanishing condition is imposed for the covariant current, which is required by the regularity of the physical quantities at the horizon. Now $\left\langle J_{(H)}^{(3) r}{ }_{t t}\right\rangle$ is obtained as

$$
\begin{aligned}
\left\langle J_{(H) t t}^{(3) r}\right\rangle=\int_{r_{+}}^{r} d r \partial_{r} & {\left[2 c_{O}^{(2)} A_{t}+c_{O}^{(1)} A_{t}(r)^{2}+\frac{1}{16} c_{O}^{(1)}\left(f f^{\prime \prime}-f^{\prime 2}\right)+\frac{1}{6 \pi} A_{t}(r)^{3}\right.} \\
& \left.-\frac{1}{96 \pi} f^{2} \partial_{r}^{2} A_{t}(r)+\frac{1}{48 \pi} f f^{\prime} \partial_{r} A_{t}(r)+\frac{1}{32 \pi}\left(f f^{\prime \prime}-f^{\prime 2}\right) A_{t}(r)\right]
\end{aligned}
$$

where $c_{O}^{(1)}$ and $c_{O}^{(2)}$ are given by

$$
\begin{aligned}
c_{O}^{(1)} & =-\frac{1}{2 \pi} A_{t}\left(r_{+}\right)=\frac{Q}{2 \pi r_{+}}, \\
c_{O}^{(2)} & =\frac{1}{4 \pi} A_{t}\left(r_{+}\right)^{2}+\frac{1}{192 \pi} f^{\prime}\left(r_{+}\right)^{2}=\frac{Q^{2}}{4 \pi r_{+}}+\frac{\kappa^{2}}{48 \pi} .
\end{aligned}
$$

They are the the fluxes of the $U(1)$ current and the EM tensor at infinity.

In the outside region, the current $J_{(O)}^{(3) r}$ tt has no anomaly. Then it is given by

$$
\left\langle J_{(O)}^{(3) r}{ }_{t t}\right\rangle=c_{O}^{(3)}+2 c_{O}^{(2)} A_{t}+c_{O}^{(1)} A_{t}(r)^{2}+\frac{1}{16} c_{O}^{(1)}\left(f f^{\prime \prime}-f^{\prime 2}\right)+\frac{1}{6 \pi} A_{t}(r)^{3},
$$

where the integration constant $c_{O}^{(3)}$ gives the value of the flux at infinity. The current is written as a sum in two regions

$$
J_{t t}^{(3) r}=J_{(O) t t}^{(3) r} \Theta_{+}(r)+J_{(H) t t}^{(3) r} H(r)
$$

where $\Theta_{+}(r)=\Theta\left(r-r_{+}-\epsilon\right)$ and $H(r)=1-\Theta_{+}(r)$ are step functions which are defined in the region $r \geq r_{+}$. Note that this current is a part of the total current because we omitted the ingoing modes near the horizon. The contribution from the ingoing modes $\left\langle K^{(3) r}{ }_{t t}\right\rangle$ is determined by imposing the following two conditions: (1) it cancels the anomalous part in the near-horizon region, (2) there is no ingoing flux from the infinity. The second 
condition is required in the Unruh vacuum. Then we have

$$
\begin{gathered}
\left\langle K_{t t}^{(3) r}\right\rangle=-\left[\frac{1}{6 \pi} A_{t}(r)^{3}-\frac{1}{96 \pi} f^{2} \partial_{r}^{2} A_{t}(r)+\frac{1}{48 \pi} f f^{\prime} \partial_{r} A_{t}(r)\right. \\
\left.+\frac{1}{32 \pi}\left(f f^{\prime \prime}-f^{\prime 2}\right) A_{t}(r)\right] H(r)
\end{gathered}
$$

Requiring the continuity of the total current at $r=r_{+}+\epsilon$, which contains both of the outgoing and ingoing modes, the asymptotic flux of the spin-3 current is obtained as

$$
c_{O}^{(3)}=-\frac{\kappa^{2}}{24 \pi} A_{t}\left(r_{+}\right)-\frac{1}{6 \pi} A_{t}\left(r_{+}\right)^{3}=\frac{\kappa^{2}}{24 \pi} \frac{Q}{r_{+}}+\frac{1}{6 \pi}\left(\frac{Q}{r_{+}}\right)^{3} .
$$

This value coincides with the $n=2$ moment of the Hawking fluxes in (B.2).

\subsection{Spin 4 gauge current}

By using a similar procedure, we can derive the flux of the spin 4 current. The current in the near-horizon region $J_{(H) t t t}^{(4) r}$ satisfies the anomaly equation (2.7) with $c_{L}=0, c_{R}=1$, and in the outside region, the current $J_{(O)}^{(4) r}$ ttt satisfies the conservation equation without the anomalies. By solving these equations with the regularity condition at the horizon, we have the following results for the expectation values of the currents $\left\langle J_{(H)}^{(4) r} t_{t t}\right\rangle$ in region $H,\left\langle J_{(O)}^{(4) r}{ }_{t t t}\right\rangle$ in region $O$, and the contribution from the ingoing modes $\left\langle K^{(4) r}{ }_{t t t}\right\rangle$ :

$$
\begin{aligned}
\left\langle J_{(H) t t t}^{(4) r}\right\rangle= & \int_{r_{+}}^{r} d r \partial_{r}\left[3 c_{O}^{(3)} A_{t}(r)+3 c_{O}^{(2)} A_{t}(r)^{2}+c_{O}^{(1)} A_{t}(r)^{3}+\frac{1}{16} c_{O}^{(2)}\left(4 f f^{\prime \prime}-5 f^{\prime 2}\right)\right. \\
& -\frac{1}{16 \pi} c_{O}^{(1)}\left(f^{2} \partial_{r}^{2} A_{t}(r)-2 f f^{\prime} \partial_{r} A_{t}(r)+\left(-4 f f^{\prime \prime}+5 f^{2}\right) A_{t}(r)\right)+\frac{1}{8 \pi} A_{t}(r)^{4} \\
& -\frac{1}{32 \pi} f^{2} \partial_{r}^{2} A_{t}(r) A_{t}(r)+\frac{1}{16 \pi} f f^{\prime} \partial_{r} A_{t}(r) A_{t}(r)+\frac{1}{64 \pi}\left(4 f f^{\prime \prime}-5 f^{\prime 2}\right) A_{t}(r)^{2} \\
& \left.+\frac{1}{30720 \pi}\left(43 f^{\prime 4}-112 f f^{\prime 2} f^{\prime \prime}+52 f^{2} f^{\prime \prime 2}+36 f^{2} f^{\prime} f^{\prime \prime \prime}-12 f^{3} f^{\prime \prime \prime \prime}\right)\right], \quad(3.11) \\
\left\langle J_{(O)}^{(4) r}{ }_{t t t}\right\rangle= & c_{O}^{(4)}+3 c_{O}^{(3)} A_{t}(r)+3 c_{O}^{(2)} A_{t}(r)^{2}+c_{O}^{(1)} A_{t}(r)^{3}+\frac{1}{16} c_{O}^{(2)}\left(4 f f^{\prime \prime}-5 f^{\prime 2}\right) \\
& -\frac{1}{16 \pi} c_{O}^{(1)}\left(f^{2} \partial_{r}^{2} A_{t}(r)-2 f f^{\prime} \partial_{r} A_{t}(r)+\left(-4 f f^{\prime \prime}+5 f^{2}\right) A_{t}(r)\right), \\
\left\langle K_{t t t}^{(4) r}\right\rangle= & -\left[\frac{1}{8 \pi} A_{t}(r)^{4}-\frac{1}{32 \pi} f^{2} \partial_{r}^{2} A_{t}(r) A_{t}(r)+\frac{1}{16 \pi} f f^{\prime} \partial_{r} A_{t}(r) A_{t}(r)\right. \\
& +\frac{1}{64 \pi}\left(4 f f^{\prime \prime}-5 f^{\prime 2}\right) A_{t}(r)^{2} \\
& \left.+\frac{1}{30720 \pi}\left(43 f^{\prime 4}-112 f f^{\prime 2} f^{\prime \prime}+52 f^{2} f^{\prime \prime 2}+36 f^{2} f^{\prime} f^{\prime \prime \prime}-12 f^{3} f^{\prime \prime \prime \prime}\right)\right] .
\end{aligned}
$$


By imposing the continuity of the total current, we have the flux $c_{O}^{(4)}$ at infinity

$$
c_{O}^{(4)}=\frac{7 \kappa^{4}}{1920 \pi}+\frac{\kappa^{2}}{16 \pi}\left(\frac{Q}{r_{+}}\right)^{2}+\frac{1}{8 \pi}\left(\frac{Q}{r_{+}}\right)^{4} .
$$

The value of the flux $c_{O}^{(4)}$ coincides with the $n=3$ moment of the Hawking flux B.2.

We have shown that the values of the anomalies for spin 3 and 4 currents at the horizon determine the Hawking fluxes at infinity. This derivation is a direct generalization of the anomaly method [3, 5] to HS fluxes. Finally we notice that since we have used the anomaly equations for HS currents constructed from fermions, these results are valid only for fermions. For other fields than fermions with a different conformal weight, the relations between holomorphic and covariant HS currents in [13] are changed and accordingly so are the anomaly equations. It is an interesting problem to classify the anomaly equations and see the universality of Hawking radiation.

\section{Christen-Fulling method for HS currents}

In this section, we show that we can also obtain the correct Hawking fluxes for the HS currents by solving the conservation equations and the trace anomalies for $c_{L}=c_{R}=1$. It is a generalization of Christensen and Fulling's method [12, in which the energy flux is obtained by solving the conservation of EM tensor and trace anomaly. In the case of charged black holes, we need to combine to solve the equations for the chiral $U(1)$ anomaly with the trace anomaly [11].

\subsection{Spins up to 4}

Now let us solve the conservation equations and trace anomaly equations summarized in section 2 for $c_{L}=c_{R}=1$. In the following we employ the conformal gauge $d s^{2}=e^{\varphi} d u d v$ for the gravitational background and the Lorenz gauge $\nabla^{\mu} A_{\mu}=0$ for the background gauge field. In these gauges, the equations are decomposed into holomorphic (i.e. independent of $v$ ) and anti-holomorphic (indep. of $u$ ) components and can be solved as

$$
\begin{gathered}
J_{u}^{(1)}=j^{(1)}(u)+\frac{1}{\pi} A_{u}, \\
J_{u u}^{(2)}=j^{(2)}(u)+2 A_{u} J_{u}^{(1)}-\frac{1}{\pi} A_{u}^{2}+\frac{1}{24 \pi}\left(\partial_{u}^{2} \varphi-\frac{1}{2}\left(\partial_{u} \varphi\right)^{2}\right),
\end{gathered}
$$




$$
\begin{aligned}
J_{u u u}^{(3)}= & j^{(3)}(u)+4 A_{u} J_{u u}^{(2)}+\frac{1}{4} \partial_{u} \varphi \partial_{u} J_{u}^{(1)}+\left(-4 A_{u}^{2}+\frac{1}{4}\left(\partial_{u}^{2} \varphi-\left(\partial_{u} \varphi\right)^{2}\right)\right) J_{u}^{(1)} \\
& -\frac{1}{6 \pi}\left(\partial_{u}^{2} \varphi-\frac{1}{2}\left(\partial_{u} \varphi\right)^{2}\right) A_{u}-\frac{1}{12 \pi} \partial_{u}^{2} A_{u}+\frac{4}{3 \pi} A_{u}^{3} \\
J_{u u u u}^{(4)}= & j^{(4)}(u)+6 A_{u} J_{u u u}^{(3)}+\frac{3}{4} \partial_{u} \varphi \partial_{u} J_{u u}^{(2)}+\left[\partial_{u}^{2} \varphi-\frac{5}{2}\left(\partial_{u} \varphi\right)^{2}-12 A_{u}^{2}\right] J_{u u}^{(2)} \\
& -\frac{3}{2} A_{u} \partial_{u} \varphi \partial_{u} J_{u}^{(1)}+\left[-\frac{3}{2} A_{u}\left(\partial_{u}^{2} \varphi-\left(\partial_{u} \varphi\right)^{2}\right)-\frac{1}{2} \partial_{u}^{2} A_{u}+8 A_{u}^{3}\right] J_{u}^{(1)} \\
& +\frac{1}{2 \pi} A_{u}\left[\partial_{u}^{2} A_{u}+\left(\partial_{u}^{2} \varphi-\frac{1}{2}\left(\partial_{u} \varphi\right)^{2}\right) A_{u}-4 A_{u}^{3}\right] \\
& -\frac{1}{160 \pi}\left(\partial_{u}^{4} \varphi-\partial_{u} \varphi \partial_{u}^{3} \varphi+\frac{4}{3}\left(\partial_{u}^{2} \varphi\right)^{2}-\frac{7}{3}\left(\partial_{u} \varphi\right)^{2} \partial_{u}^{2} \varphi+\frac{7}{12}\left(\partial_{u} \varphi\right)^{4}\right) .
\end{aligned}
$$

Here $j^{(n)}(u)$ are arbitrary holomorphic functions, which can be fixed by boundary conditions at the horizon.

First, we consider the flux of the $U(1)$ current $\left\langle J_{u}^{(1)}\right\rangle$ in the black hole background. We impose the regularity condition that $\left\langle J_{U}^{(1)}\right\rangle$ is regular at the horizon $U=0$ in the Kruskal coordinate (A.8). This condition corresponds to taking the Unruh vacuum. The current $J_{u}^{(1)}$ in the $(u, v)$ coordinates and another $J_{U}^{(1)}$ in the Kruskal $(U, V)$ coordinates are related by

$$
J_{U}^{(1)}=-\frac{1}{\kappa U} J_{u}^{(1)}
$$

Therefore the regularity condition for $\left\langle J_{U}^{(1)}\right\rangle$ means $\left\langle J_{u}^{(1)}\right\rangle \rightarrow 0$ at the horizon. Since $J_{u}^{(1)}$ is written as

$$
J_{u}^{(1)}=j^{(1)}(u)+\frac{e^{2}}{\pi} A_{u},
$$

the condition is equivalent to the following boundary condition for the holomorphic current $j^{(1)}(u)$,

$$
\left\langle j^{(1)}(u)\right\rangle \stackrel{r \rightarrow r_{+}}{\longrightarrow}-\left.\frac{e^{2}}{\pi} A_{u}\right|_{r=r_{+}}=\frac{e^{2} Q}{2 \pi r_{+}} .
$$

Besides, the Reissner-Nordström black hole solution is static and thus $\left\langle j^{(1)}(u)\right\rangle$, which is a holomorphic function of $u=t-r_{*}$, should be a constant, namely $\left\langle j^{(1)}(u)\right\rangle=e^{2} Q / 2 \pi r_{+}$. Therefore the flux at infinity is obtained as

$$
\left\langle J_{u}^{(1)}\right\rangle \stackrel{r \rightarrow \infty}{\longrightarrow}\left\langle j^{(1)}(u)\right\rangle=\frac{e^{2} Q}{2 \pi r_{+}} .
$$


This value is equal to the $n=0$ result in (B.2).

Similarly, we can derive fluxes of the higher-spin currents by imposing the regularity condition that the currents in the Kruskal coordinate are regular at the horizon. Also it is important that the expectation value of the covariant HS current $\left\langle J_{u \cdots u}^{(n)}\right\rangle$ coincides with the value of the conformal HS current $\left\langle j^{(n)}(u)\right\rangle$ at infinity because contributions from the background fields approach to zero there. The results for the HS currents up to spin 4 are given as follows:

$$
\begin{aligned}
\left\langle J_{u u}^{(2)}\right\rangle & \stackrel{r \rightarrow \infty}{\longrightarrow}\left\langle j^{(2)}(u)\right\rangle=\frac{\kappa^{2}}{48 \pi}+\frac{1}{4 \pi}\left(\frac{e Q}{r_{+}}\right)^{2}, \\
\left\langle J_{u u u}^{(3)}\right\rangle & \stackrel{r \rightarrow \infty}{\longrightarrow}\left\langle j^{(3)}(u)\right\rangle=\frac{\kappa^{2}}{24 \pi} \frac{e Q}{r_{+}}+\frac{1}{6 \pi}\left(\frac{e Q}{r_{+}}\right)^{3}, \\
\left\langle J_{\text {uuuu }}^{(4)}\right\rangle & \stackrel{r \rightarrow \infty}{\longrightarrow}\left\langle j^{(4)}(u)\right\rangle=\frac{7 \kappa^{4}}{1920 \pi}+\frac{\kappa^{2}}{16 \pi}\left(\frac{e Q}{r_{+}}\right)^{2}+\frac{1}{8 \pi}\left(\frac{e Q}{r_{+}}\right)^{4} .
\end{aligned}
$$

These values coincide with those in eq. (B.2). We have defined the currents to be totally symmetric, and their trace anomalies vanish at $r \rightarrow \infty$. Hence the fluxes of currents at infinity are given by $J_{t \cdots t}^{(n) r}=J_{u \cdots u}^{(n)}-J_{v \cdots v}^{(n)}$. In the Unruh vacuum, the ingoing modes vanish there and we have $J_{t \cdots t}^{(n) r}=J_{u \cdots u}^{(n)}$.

We have now shown that, by solving the conservation equations and the trace anomalies up to spins 4, we can reproduce the correct results of higher-moments of the Hawking fluxes.

\subsection{General higher spins}

For spins up to 4, we solved the conservation equations and trace anomalies in section 2 and obtained equations (4.1)-(4.4). There holomorphic functions $j^{(n)}(u)$ have been introduced in solving the equations in the form of $\partial_{v}(\cdots)=0$. They can be determined by imposing appropriate boundary conditions for the currents. In conformal field theories, these holomorphic functions $j^{(n)}(u)$ play an important role as generators of conformal $W_{1+\infty}$ transformations which are HS generalizations of conformal transformations. We call them holomorphic HS currents. In [13, we have derived a relation between these holomorphic HS currents and the original covariant HS currents as an identity of their generating functions. Actually, the gauge and trace anomalies for spin 3 and 4 in section 2 were derived from these relations. In this sense, solving the conservation equations and trace anomalies is equivalent to the relation between the holomorphic and covariant 
HS currents. In this subsection, we use the relation to derive all the higher moments of Hawking fluxes.

We first summarize the relation. See [13] for details. A generating function of the holomorphic HS currents is defined as

$$
G_{h o l}(u+\alpha, u-\alpha) \equiv \sum_{n=0}^{\infty} \frac{(2 i \alpha)^{n}}{n !} j^{(n+1)}(u)=\Psi^{\dagger}(u+\alpha) \Psi(u-\alpha)+\frac{i}{4 \pi \alpha} .
$$

Here $\Psi(u)$ is a holomorphic fermion field, which is a holomorphic function of $u$ in the electric and gravitational background and related to the original fermion field $\psi(u, v)$. Each HS current should be regularized appropriately and (4.12) should be considered as a formal power series of $\alpha$.

By using the definition of the holomorphic fermion field $\Psi(u)$, this generating function can be written as

$$
\begin{aligned}
G_{h o l}(u+\alpha, u-\alpha)= & e^{\frac{1}{2}(\varphi(u+\alpha)+\varphi(u-\alpha))-2 i(\eta(u+\alpha)-\eta(u-\alpha))} \\
& \times\left[e^{-\frac{1}{4}(\varphi(u+\alpha)+\varphi(u-\alpha))+i(\eta(u+\alpha)-\eta(u-\alpha))} \psi^{\dagger}(u+\alpha, v) \psi(u-\alpha, v)\right. \\
& \left.\quad+\frac{i \hbar}{2 \pi} \frac{1}{x(u+\alpha)-x(u-\alpha)}\right] \\
& -\frac{i \hbar}{2 \pi} \frac{e^{\frac{1}{2}(\varphi(u+\alpha)+\varphi(u-\alpha))-2 i(\eta(u+\alpha)-\eta(u-\alpha))}}{x(u+\alpha)-x(u-\alpha)}+\frac{i \hbar}{4 \pi \alpha} .
\end{aligned}
$$

Here we formally introduced a new coordinate $x$ which satisfy $\partial_{x}=e^{-\varphi(u, v)} \partial_{u}$ and regard $x$ as a function of $u$ (i.e., $v$ is kept fixed). $\varphi(u, v)$ is the conformal factor of the gravitational background and $\eta(u, v)$ represents the electric background in the Lorentz gauge, $A_{u}=$ $\partial_{u} \eta(u, v)$. The first term in (4.13) with a bracket $[\cdots]$ can be written in terms of the regularized covariant HS currents and their derivatives. The second and third terms give quantum corrections. By expanding the r.h.s. of (4.13) with respect to the parameter $\alpha$, we can obtain the equations relating the holomorphic HS currents with the sum of the covariant HS currents and quantum corrections:

$$
\sum_{n=0}^{\infty} \frac{(2 i \alpha)^{n}}{n !}\left(J_{u \cdots u}^{(n+1)}+\sum_{m, k=0}^{n-1} C_{m k}^{(n+1)}\left(\varphi, A_{u}\right) \partial_{u}^{k} J_{u \cdots u}^{(m+1)}+\hbar D^{(n+1)}\left(\varphi, A_{u}\right)\right)
$$

where $C_{m k}\left(\varphi, A_{u}\right)$ and $D^{(n+1)}\left(\varphi, A_{u}\right)$ are functions of $\varphi$ and $A_{u}$ which vanish at infinity. By comparing the coefficients of $\alpha^{n}$ up to $n=3$, we get the equations in (4.1)-(4.4). Hence 
the above relation contains the full information of the anomalies. We use it to evaluate the fluxes of the HS currents than spin 4.

Let us calculate the Hawking fluxes. First we evaluate the expectation value of (4.13) in the near horizon region. We impose the regularity condition for the covariant currents at the horizon; $J_{u \cdots u}^{(n)} \rightarrow 0$. Then the first term in the r.h.s. of (4.13) becomes 0 . Thus we need to evaluate the last two terms, which are proportional to $\hbar$.

In order to evaluate these quantum contributions, we first note that $\partial_{u}=-f \partial_{r} / 2$ and $f \rightarrow 0$ near the horizon (see the appendix $\mathrm{A}$ ). Then we have, near the horizon $u \rightarrow \infty$, $\eta(u+\alpha)-\eta(u-\alpha) \rightarrow 2 \alpha \partial_{u} \eta=2 \alpha A_{u}\left(r_{+}\right)=-\alpha Q / r_{+}$. Higher derivatives $\partial_{u}^{n} \eta$ for $n>1$ vanish at the horizon. Because of the same reason, we have $\varphi(u+\alpha)+\varphi(u-\alpha) \rightarrow 2 \varphi(u)$ and

$$
\begin{aligned}
& x(u+\alpha)-x(u-\alpha)=2 \sum_{n=0}^{\infty} \frac{\alpha^{2 n+1}}{(2 n+1) !} \partial_{u}^{2 n+1} x \\
& \rightarrow 2 \sum_{n=0}^{\infty} \frac{\alpha^{2 n+1}}{(2 n+1) !}\left(\partial_{u} \varphi\right)^{2 n} e^{\varphi}=2 \frac{e^{\varphi}}{\kappa} \sinh (\alpha \kappa)
\end{aligned}
$$

where $\kappa$ is the surface gravity at the horizon; $\partial_{u} \varphi(u)=\partial_{u} \log f \rightarrow-\kappa$. As a result, we obtain

$$
\left\langle G_{h o l}(u+\alpha, u-\alpha)\right\rangle=\sum_{n=0}^{\infty} \frac{(2 i \alpha)^{n}}{n !}\left\langle j^{(n+1)}(u)\right\rangle \rightarrow-\frac{i \hbar}{4 \pi \alpha} \frac{\alpha \kappa e^{-\frac{2 i \alpha Q}{r_{+}}}}{\sinh (\alpha \kappa)}+\frac{i \hbar}{4 \pi \alpha} .
$$

By expanding this equation with respect $\alpha$ and comparing their coefficients, we can obtain the expectation value $\left\langle j^{(n)}(u)\right\rangle$ which are equal to the value of the covariant current $\left\langle J_{u \cdots u}^{(n)}\right\rangle$ at infinity. We can show that these values of the fluxes coincide with (B.2). Besides, as is pointed out in [10, 11], this generating function is the regular part of the fermion Green function, and (4.16) is interpreted as the temperature-dependent part of the thermal Green function with chemical potential. It means that the thermal excitation of the fields are introduced through the quantum effect.

By this analysis, we can also see, which was implicitly used in [10, 11], that the fluxes of the covariant HS currents corresponding to the physical quantities are equal to those of the holomorphic currents at infinity, since both values of $\varphi$ and $A_{u}$ asymptotically decay to zero. 


\section{Summary}

In this paper, by using the higher-spin (HS) generalizations of the gauge and trace anomaly equations, we reproduced the HS fluxes of Hawking radiation.

We first used the anomaly equations for spin 3 and 4 currents to derive the Hawking fluxes and reproduced the correct values. This is a direct HS generalization of the gauge and gravitational anomaly method by Robinson and Wilczek [3] and by some of us [5].

We then solved the conservation equations and trace anomalies for the HS currents. This again reproduces the correct values of Hawking fluxes for spin 3 and 4 . This is now considered as a HS generalization of the method by Christensen and Fulling [12]. We further employed the generating function of the general HS currents and derived the complete Fermi-Dirac distribution.

Finally we should notice the following. The anomaly equations we have used are constructed from the fermion currents, and they are modified for other fields with different conformal weights. This gives a different value of HS fluxes of Hawking radiation for bosons.

\section{A Reissner-Nordström black hole}

We summarize the basics of Reissner-Nordström black holes. The metric and the gauge potential of Reissner-Nordström black holes with mass $M$ and charge $Q$ are given by

$$
\begin{aligned}
d s^{2} & =f(r) d t^{2}-\frac{1}{f(r)} d r^{2}-r^{2} d \Omega_{2}^{2} \\
A_{t} & =-\frac{Q}{r}
\end{aligned}
$$

where

$$
f(r)=1-\frac{2 M}{r}+\frac{Q^{2}}{r^{2}}=\frac{\left(r-r_{+}\right)\left(r-r_{-}\right)}{r^{2}}
$$

and the radius of outer (inner) horizon $r_{ \pm}$is given by

$$
r_{ \pm}=M \pm \sqrt{M^{2}-Q^{2}}
$$

It is useful to define the tortoise coordinate by solving $d r_{*}=d r / f$ as

$$
r_{*}=r+\frac{1}{2 \kappa_{+}} \ln \frac{\left|r-r_{+}\right|}{r_{+}}+\frac{1}{2 \kappa_{-}} \ln \frac{\left|r-r_{-}\right|}{r_{-}} .
$$


Here the surface gravity at $r_{ \pm}$is given by

$$
\kappa_{ \pm}=\frac{1}{2} f^{\prime}\left(r_{ \pm}\right)=\frac{r_{ \pm}-r_{\mp}}{2 r_{ \pm}^{2}} .
$$

In the following we consider a region near the outer horizon. First we define the light-cone coordinates, $u=t-r_{*}$ and $v=t+r_{*} \cdot u(v)$ are the outgoing (ingoing) coordinates and the metric in these coordinates becomes as

$$
d s^{2}=f\left(d t^{2}-d r_{*}^{2}\right)-r^{2} d \Omega^{2}=f d u d v-r^{2} d \Omega^{2}
$$

In order to investigate the physics near the outer horizon, since $(u, v)$ coordinate is still singular at the horizon, it is important to introduce a regular coordinate, Kruskal coordinate, defined by the transformations

$$
U=-e^{-\kappa_{+} u}, \quad V=e^{\kappa_{+} v}
$$

The metric becomes

$$
d s^{2}=\frac{r_{+} r_{-}}{\kappa_{+}^{2}} \frac{1}{r^{2}} e^{-2 \kappa_{+} r}\left(\frac{r_{-}}{r-r_{-}}\right)^{\frac{\kappa_{+}}{\kappa_{-}}-1} d U d V-r^{2} d \Omega^{2} .
$$

If we restrict to see the two-dimensional $(r, t)$ section, both of these coordinates (A.7), (A.9) have the forms of the conformal gauge

$$
d s^{2}=e^{\varphi(u, v)} d u d v=e^{\varphi^{\prime}(U, V)} d U d V
$$

The transformation (A.8) is a conformal transformation from an asymptotically flat coordinate to a regular coordinate near the horizon.

In the bulk of the paper we omit the subscript + of the surface gravity $\kappa_{+}$at the outer horizon because $\kappa_{-}$does not appear in our analysis.

\section{B Higher moments of Hawking fluxes}

In this appendix, we calculate the higher-spin fluxes of the Fermi-Dirac distribution with Hawking temperature and the chemical potential. We first note that two-dimensional effective theories near the horizon in various black holes have the same form as that in charged black holes. It is thus enough to consider Hawking fluxes in the ReissnerNordström black hole. Some basic facts of the Reissner-Nordström black hole are sum- 
marized in the appendix $\mathrm{A}$. Thermal radiation of fermions with charge $q$ from ReissnerNordström black hole satisfies the Fermi-Dirac distribution $N_{q}(\omega)$ with a chemical potential corresponding to the value of the electric potential at the horizon,

$$
N_{q}(\omega)=\frac{1}{e^{\beta\left(\omega-\frac{q Q}{r_{+}}\right)}+1} .
$$

Here $\beta=\frac{2 \pi}{\kappa}$ is the inverse temperature. From this distribution function, we can evaluate the thermal fluxes of higher-spin currents at infinity as

$$
\begin{aligned}
\int_{0}^{\infty} & \frac{d \omega}{2 \pi}\left[\omega^{n} N_{e}(w)-(-\omega)^{n} N_{-e}(w)\right] \\
& =\sum_{m=1}^{\left\lceil\frac{n}{2}\right\rceil}\left(\frac{e Q}{r_{+}}\right)^{n+1-2 m} \frac{n !\left(1-2^{1-2 m}\right) B_{m} \kappa^{2 m}}{2 \pi(2 m) !(n-2 m+1) !}+\frac{1}{2 \pi(n+1)}\left(\frac{e Q}{r_{+}}\right)^{n+1} .
\end{aligned}
$$

Here $B_{m}$ is the Bernoulli number $\left(B_{1}=1 / 6, B_{2}=1 / 30\right)$ and $\lceil x\rceil$ is the ceiling function, which returns the smallest integer not less than $x$. This integral is the $n$-th moment of the thermal flux containing contributions from the fermion with charge $e$ and its antiparticle with charge $-e$.

\section{References}

[1] S. Hawking, "Particle Creation By Black Holes," Commun. Math. Phys. 43, 199 (1975).

[2] S. Hawking, "Black Hole Explosions," Nature (London) 248, 30 (1974).

[3] S. P. Robinson and F. Wilczek, "A relationship between Hawking radiation and gravitational anomalies," Phys. Rev. Lett. 95, 011303 (2005). arXiv:gr-qc/0502074.

[4] W. Unruh, "Notes On Black Hole Evaporation," Phys. Rev. D 14, 870 (1976).

[5] S. Iso, H. Umetsu and F. Wilczek, "Hawking radiation from charged black holes via gauge and gravitational anomalies," Phys. Rev. Lett. 96, 151302 (2006) arXiv:hep-th/0602146.

[6] S. Iso, H. Umetsu and F. Wilczek, "Anomalies, Hawking radiations and regularity in rotating black holes," Phys. Rev. D 74, 044017 (2006) arXiv:hep-th/0606018. 
[7] K. Murata and J. Soda, "Hawking radiation from rotating black holes and gravitational anomalies," Phys. Rev. D 74, 044018 (2006) arXiv:hep-th/0606069.

[8] S. Iso, T. Morita and H. Umetsu, "Quantum anomalies at horizon and Hawking radiations in Myers-Perry black holes," JHEP 0704, 068 (2007) arXiv:hep-th/0612286.

[9] E. C. Vagenas and S. Das, JHEP 0610, 025 (2006) arXiv:hep-th/0606077]; M. R. Setare, Eur. Phys. J. C 49, 865 (2007) arXiv:hep-th/0608080]; Z. Xu and B. Chen, Phys. Rev. D 75, 024041 (2007) arXiv:hep-th/0612261; Q. Q. Jiang and S. Q. Wu, Phys. Lett. B 647, 200 (2007) arXiv:hep-th/0701002]; Q. Q. Jiang, S. Q. Wu and X. Cai, Phys. Lett. B 651, 58 (2007) arXiv:hep-th/0701048; Q. Q. Jiang, S. Q. Wu and X. Cai, Phys. Rev. D 75, 064029 (2007) [Erratum-ibid. 76, 029904 (2007)] arXiv:hep-th/0701235]; X. Kui, W. Liu and H. Zhang, Phys. Lett. B 647, 482 (2007) arXiv:hep-th/0702199; H. Shin and W. Kim, JHEP 0706, 012 (2007) arXiv:0705.0265 [hep-th]]; J. J. Peng and S. Q. Wu, arXiv:0705.1225 [hep-th]; Q. Q. Jiang, Class. Quant. Grav. 24, 4391 (2007) arXiv:0705.2068 [hepth]]; B. Chen and W. He, arXiv:0705.2984 [gr-qc]; U. Miyamoto and K. Murata, arXiv:0705.3150 [hep-th]; Q. Q. Jiang, S. Q. Wu and X. Cai, Phys. Lett. B 651, 65 (2007) [arXiv:0705.3871 [hep-th]]; S. Q. Wu and J. J. Peng, arXiv:0706.0983 [hep-th]; W. Kim and H. Shin, JHEP 0707, 070 (2007) [arXiv:0706.3563 [hep-th]]; Z. Z. Ma, arXiv:0709.3684 [hep-th]; K. Murata and U. Miyamoto, arXiv:0707.0168 [hep-th]; R. Banerjee and S. Kulkarni, arXiv:0709.3916 [hep-th].

[10] S. Iso, T. Morita and H. Umetsu, "Higher-spin currents and thermal flux from Hawking radiation," Phys. Rev. D 75, 124004 (2007) arXiv:hep-th/0701272.

[11] S. Iso, T. Morita and H. Umetsu, "Fluxes of Higher-spin Currents and Hawking Radiations from Charged Black Holes," Phys. Rev. D 76, 064015 (2007) arXiv:0705.3494 [hep-th]].

[12] S. Christensen and S. Fulling, "Trace Anomalies And The Hawking Effect," Phys. Rev. D 15, 2088 (1977).

[13] S. Iso, T. Morita and H. Umetsu, "Higher-spin Gauge and Trace Anomalies in Twodimensional Backgrounds," arXiv:0710.0453 [hep-th]. 
[14] K. Fujikawa and H. Suzuki, Path integrals, and quantum anomalies (Oxford Science Publications, Oxford, 2004)

[15] R. Bertlmann, Anomalies in Quantum Field Theory (Oxford Science Publication, Oxford, 2000)

[16] To an observer outside a black hole, gravity in the near-horizon region has twodimensional conformal symmetries. This fact is stressed in the following references; S. Carlip, "Black hole entropy from conformal field theory in any dimension," Phys. Rev. Lett. 82, 2828 (1999) arXiv:hep-th/9812013; S. N. Solodukhin, "Conformal description of horizon's states," Phys. Lett. B 454, 213 (1999) arXiv:hep-th/9812056);

[17] R. Banerjee and S. Kulkarni, "Hawking Radiation and Covariant Anomalies," arXiv:0707.2449 [hep-th]. 